\title{
Preface: meshfree and particle methods for modeling extreme loadings
}

\author{
J. S. Chen ${ }^{1} \cdot$ Sheng-Wei Chi ${ }^{2}$ - $\cdot$ Mike Hillman ${ }^{3}$ (])
}

Published online: 17 November 2019

(c) OWZ 2019

\section{In memory of Steve Attaway (1960-2019)}

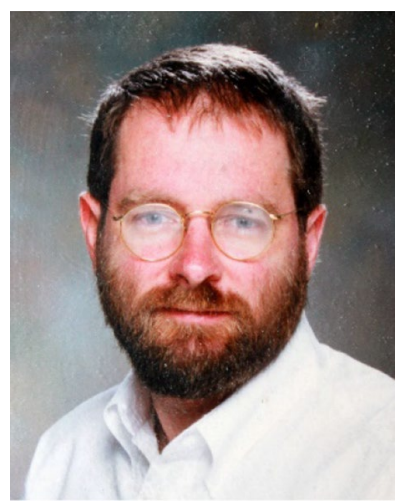

This special issue is dedicated to Steve Attaway, who passed away on February 28, 2019. Steve Attaway worked at Sandia National Laboratories in Albuquerque, NM, for over 30 years making significant contributions in highperformance computing, shock physics, meshfree methods, the geosciences, concrete mechanics, and blast effects on structures. Steve's early contributions in meshfree methods included stability analysis of smoothed particle hydrodynamics (SPH) (Swegle, J., Hicks, D., and Attaway, S., 1995, "Smoothed Particle Hydrodynamics Stability Analysis," JCP v. 116) and the coupling of SPH with the finite

J. S. Chen

js-chen@eng.ucsd.edu

Sheng-Wei Chi

swchi@uic.edu

Mike Hillman

mhillman@psu.edu

University of California, San Diego, La Jolla, USA

2 University of Illinois at Chicago, 945 W Taylor St., Chicago, IL 60608, USA

3 The Pennsylvania State Univeristy, 201 Old Main, University Park, PA 16802, USA element method in a massively parallel explicit dynamics framework (Attaway, S., Heinstein, M., and Swegle, J., 1994, "Coupling of smooth particle hydrodynamics with the finite element method," Nuclear Eng. Design, v. 150). Steve understood the great potential of meshfree methods for modeling structures under extreme loading conditions including blast effects, penetration, and perforation. Steve helped bring big, seemingly impossible ideas into realization, from parallelizing the PRONTO FEA code in the early 1990s to pushing shock physics codes to run on hundreds of thousands of cores on the world's fastest supercomputers. Throughout his career, he was recognized with awards and accolades from the Department of Energy. And yet, Steve was never focused solely on the awards, accolades, recognitions, or technical accomplishments, significant though they were. He also invested his time in training the next generation of big thinkers and problem solvers. He probably spent as much time mentoring those around him as he did relishing every opportunity to tackle the "big problems" brought to Sandia for solving. The impact and legacy of Steve Attaway was not just about his technical achievements, but how he was always willing to help, instruct, guide, direct, lead, and serve others (contributed by Joseph Bishop, Sandia National Laboratories, Albuquerque, NM).

\section{Summary}

In the field of extreme events research, physical experimentation is often limited due to the high rates, short time scales, large deformation response, and/or physical sizes that are inherently present in these problems. Computation extends our capabilities beyond these experimental limitations; however, methods suitable for modeling these events must be versatile and stable when dealing with rough solutions in the form of transient strong discontinuities (fractures) and weak discontinuities (localization), severe material deformation and material instability, multi-body contact without 
a priori knowledge of potential contacting bodies and contact surfaces, multiple and evolving length scales, multiple and coupled physics, among other features. Meshfree and particle methods offer unique features that are particularly attractive for modeling extreme events. These methods have the potential to relieve issues related to mesh distortion and entanglement, and allow arbitrary smoothness (and roughness) in the approximation to be varied in space and time. This Thematic Issue aims to summarize the most recent advances in numerical methods for modeling extreme loadings and events.

The manuscript by $\mathrm{Wu}, \mathrm{Wu}, \mathrm{Lyu}, \mathrm{Pan}$, and $\mathrm{Hu}$ introduces a novel velocity smoothing technique to achieve stabilized solutions in the Galerkin-based meshfree method when the variational equation is nodally integrated. The nodal state variables at a node, e.g., nodal mass, density, and linear momentum, are gathered from the neighboring nodes and smoothed using the shape functions associated with the nodes. The smoothing technique was shown to conserve the nodal mass and linear momentum, and it does not require an additional stabilization term nor a tunable parameter. The proposed method is shown to demonstrate stable results in severe deformation simulations including flow-drill-screw thread forming processes.

Although the meshfree shape function can generally be constructed to possess desired consistency and continuity, its direct derivative computation is costly. The manuscript by Wu, Wang, Lin, and Qi proposes a smoothed gradient reproducing kernel formulation for solutions of the fourth-order phase field model for brittle fracture, where the second-order gradient is required in the Galerkin formulation. The firstorder gradient in the method is constructed by enforcing the integration consistency condition over a smoothing kernel; then, the second-order gradient is obtained by direct differentiation of the first-order smoothed gradient. With triangular background cells for domain integration, the proposed method shows good accuracy and computational efficiency for solving the phase field model.

Yamaguchi, Takase, Moriguchi and Terada present a coupled hydromechanical material point method (MPM) formulation for fluid-saturated porous media. A fractional-step time integration method is introduced to eliminate instabilities associated with incompressibility; meanwhile, the solid skeleton and fluid are discretized independently by material points, which allows free-surface flows in both fluid and solid. B-Spline basis functions are further introduced to circumvent cell-crossing instabilities in traditional MPM. The method is benchmarked by several well-selected examples, including consolidation and slope stability analysis, and an experimental validation problem with transition of soil to a flowing mixture, with good agreement with analytical solutions and experimental data.
The manuscript by Su, Tao, Jiang, Chen, and Lu presents a fully coupled thermodynamic fluid-structure interaction (FSI) scheme with MPM discretization. FSI is naturally handled by the use of material points representing both fluids and solids separately. Meanwhile, the heat conduction equation is also treated in an MPM manner, and ultimately, thermal strains are computed at each material point to account for the thermo-mechanical response. To avoid cell-crossing instability, an ad hoc technique is adopted from previous works, which is both effective and more efficient than treatments such as the generalized interpolation material point method. Several benchmarks are solved showing good agreement with available solutions for thermo-mechanical problems and fluid-structure interaction problems. A coupled thermodynamic FSI problem is also studied, and interesting phenomenon is observed with respect to the interplay between thermal gas pressure waves and mechanical deformation of a simply supported beam.

Fluid-structure interaction problems involving the failure of solids remain a challenge for mesh-based methods due to the evolving topology involved; meanwhile, meshfree methods can overcome many associated difficulties naturally. The manuscript by Liu, Yan, and Li presents a coupling scheme for updated Lagrangian particle hydrodynamics (ULPH) and bond-based peridynamics to address this issue and is employed to model the interaction between fracturing ice and seawater. The water is modeled using the ULPH formulation with a non-local discretization of the weakly compressible Navier-Stokes equations, which naturally tracks free surfaces. Meanwhile, bond-based peridynamics is employed to model ice fracture. Coupling is performed using dummy particle techniques of previous works. They present a validation of the combined method, and a convincing demonstration problem coupling fluid, solid fracture, and a rigid body impactor.

It is well known that meshfree methods offer advantages over mesh-based methods in Lagrangian discretizations with large deformations. In the manuscript by Bishop, a comprehensive study is presented on the accuracy of these two discretizations in representing large deformation kinematics. A manufactured solution is considered with the norm of the Lagrange strains approaching 10. Uniform and increasingly randomly perturbed discretizations are considered as well. The finite element approximation consistently shows higher errors than meshfree approximations and, in cases of severe perturbation, was not computable. Overall, this work provides a compelling argument for the use of meshfree methods over mesh-based methods in Lagrangian solid mechanics involving large deformations.

Trask and Kuberry present a generalized moving least squares (GMLS) primary/dual discretization of $H$ (div) problems on surfaces. This method is a reinterpretation of previous work as a GMLS method, allowing an extension 
to higher-order accuracy and staggered grids, while also endowing the method with the approximation theory of GMLS. The method works in the coordinates of the manifold and results in a stencil discretization of the surface PDE. The proposed discretization is demonstrated to be particularly advantageous in the presence of discontinuous problem coefficients, offering stability out of the box, and algebraic convergence of fluxes, with properties parallel to div-compatible mesh-based schemes.

Mitsume, Yamada, and Yoshimura present work on the moving particle semi-implicit method, which is a Lagrangian meshfree method well suited for free-surface fluid flow problems. An efficient parallel implementation of the method is provided, which employs domain decomposition and dynamic load balancing algorithms, key aspects for an efficient and scalable parallel meshfree method implementation. This work further introduces explicitly described polygons for representing boundary walls, circumventing the need for the conventional meshfree treatment by wall particles, which has a high computational cost and problems with particle leakage. Large-scale benchmarks are solved including a validation example and a complex demonstration problem.

The work by Liu and Sun overcomes the difficulty of imposing boundary conditions on an object of a complex geometry in MPM, by mapping the true boundary to a surrogate boundary on background cells. With the implicitly represented boundary by a signed distance function and a consistent derivation, the proposed method captures arbitrary boundaries without additional degrees of freedom and provides consistent responses of materials undergoing finite deformations in the implicit MPM framework. A sand grain model that is constructed from micro-CT images was used to demonstrate the potential capability of the method in problems with complicated microstructures.

Despite extended effort, an effective technique to resolve incompressibility issues in variational-based meshfree methods remains in demand. Moutsanidis, Koester, Tupek, Chen, and Bazilevs presents work that generalizes the idea of projecting the dilatational part of a proper deformation measure onto lower-order approximations to derive the corresponding $\overline{\mathbf{B}}$ and $\overline{\mathbf{F}}$ approaches for meshfree and particle methods. The projection operators and $\overline{\mathbf{F}}$ computations were tailor-made for the conforming reproducing kernel method and immersed-particle methods. The numerical results from the proposed method demonstrate reduced stress oscillations and solutions free of volumetric locking in benchmark problems.

The manuscript by Huang, Chen, Wei, Roth, Sherburn, Bishop, Tupek, and Fang presents a meshfree implementation of the monotonic upwind scheme for conservation laws (MUSCL) method for solving Eulerian conservation laws involving strong shocks. The Rankine-Hugoniot jump conditions and entropy solution are embedded in the solution under the stabilized conforming nodal integration (SCNI) framework; meanwhile, convective transport instabilities are handled by a modified upwind scheme. The linear reconstruction with oscillation limiter introduced provides high accuracy with monotonic solutions, and the overall scheme is free of tunable parameters. Several complex shock benchmarks are solved, demonstrating the need for the combined techniques and effectiveness of the method, and good validation against experimental data is achieved.

The manuscript by Mahdavi, Chi, and Atif details a displacement-pressure formulation for saturated porous media under the reproducing kernel particle method (RKPM) framework. To capture the severe deformation in the penetration simulation, both displacement and pressure fields are approximated by semi-Lagrangian reproducing kernel approximations, in which the nodal point of the reproducing kernel follows material deformation whereas the kernel support is independent of deformation. In conjunction with modified stabilized non-conforming nodal integration, the numerical solutions are stable and accurate, when equalorder approximations are used for displacement and pressure fields, and show good agreement with experimental data from the literature.

Peridynamics has been introduced to effectively tackle dynamic problems with strong discontinuities. The treatment of shock waves in peridynamics, however, remains limited. Zhou and Hillman introduce the Godunov scheme into the state-based peridynamics for shock handling. The Riemann solution is enforced at the middle of each point pair with a surface correction derived from the equivalence between the discrete peridynamics force and the finite volume nodal force. The effectiveness of the proposed technique is promising as demonstrated in impact benchmarks, and the framework can be generally extended to other meshfree methods.

The gradient discontinuities in conventional MPM lead to the cell-crossing instability, and inaccuracy. The paper by Song, Liu, and Zhang proposes a transport point method which employes moving least squares shape functions to reconstruct state and field quantities at desired nodal points and Gauss points for integration of the weak form on the background grid. A transport point rearrangement algorithm is proposed for adaptive transport point addition, relocation, or removal for proper interpolation of state and field quantities to the grid points and for elimination of numerical fracture. Different quadrature rules were also employed for the internal cells and boundary cells for enhanced accuracy.

Despite numerous advancements made in Galerkin-based meshfree methods, an open-source code remains inaccessible to general users. The manuscript by Huang, Wei, Chen, and Hillman presents an open-source code for RKPM with incorporation of a handful of efficient and robust domain integration techniques. The common issues encountered in 
the meshfree formulation are discussed and the data structure for efficient implementation is provided in the manuscript. The open source code indeed can serve as an introduction of RKPM for newcomers and as a test bed for further development of advanced algorithms.

Hillman, Pasetto, and Zhou present a unifying framework for state-based peridynamics based on the correspondence principle (SBP-CP), and the implicit gradient reproducing kernel approximation. The relationship between these two methods is given explicitly, and contrary to previous speculations, it is shown that the non-local deformation gradient in SBP-CP is not equivalent to the implicit gradient. A unified formulation is then given, where both the implicit gradient and the non-local deformation gradient can be recovered, which is termed the reproducing kernel peridynamic approximation. This formulation also generalizes non-local approximations to derivatives beyond first-order differentiation and first-order accuracy. It is then utilized to attain higher-order accuracy than the original SBP-CP formulation, which is shown not to converge in non-uniform discretizations. The proposed method is tested in the commonly used nodal collocation setting, demonstrating convergence with and without the use of ghost boundary nodes, and in both uniform and non-uniform discretizations.

Publisher's Note Springer Nature remains neutral with regard to jurisdictional claims in published maps and institutional affiliations. 